\title{
Candida albicans urinary tract infection in a Shih Tzu dog with immune-mediated hemolytic anemia
}

\author{
In-Sung Jang ${ }^{1}$, Won-Kyoung Yoon ${ }^{1,2}$, Changbaig Hyun ${ }^{1, *}$ \\ ${ }^{1}$ Section of Small Animal Internal Medicine, College of Veterinary Medicine, Kangwon National University, \\ Chuncheon 24341, Korea \\ ${ }^{2}$ Guardian Angel Veterinary Hospital, Anyang 14112, Korea
}

(Received: February 4, 2017; Revised: April 25, 2017; Accepted: June 5, 2017)

\begin{abstract}
An 8-year-old castrated male Shih Tzu dog (weighing $7.0 \mathrm{~kg}$ ) presented with anemia and lethargy. Initial diagnosis indicated immune-mediated hemolytic anemia. During therapy, a secondary urinary infection, probably due to the immune suppressive therapy, was diagnosed. Subsequent diagnostic tests, including urinalysis and urine culture, indicated candidal cystitis. Despite ketoconazole therapy for candidal cystitis, the dog died suddenly. A Candida albicans infection was confirmed upon postmortem evaluation. Prolonged immunosuppressive therapy might be the cause of this infection. This is the first case report describing a Candida albicans urinary tract infection accompanied by hemolytic anemia in a dog in Korea.
\end{abstract}

Keywords: Candida albicans, dog, candidal cystitis, immune-mediated hemolytic anemia, urinary tract infection

Candida (C.) albicans is an opportunistic fungal pathogen that causes urogenital infections in dogs, although it is typically part of the normal flora in the gastrointestinal, urogenital, and upper respiratory tracts in dogs $[9,11]$. The contributing factors involved in a $C$. albicans infection in dogs include immunosuppressive diseases, long-term steroid therapy, mucosal damages, and long-term catheterization in the lower urinary system. Although a $C$. albicans urinary tract infection (UTI) has been well documented in dogs and cats [3, 4, 9], a UTI associated with immune-medicated hemolytic anemia (IMHA) has never been reported in dogs. This case report described a C. albicans UTI in a dog, which occurred because of IMHA and a subsequent immunosuppressive therapy.

An 8-year-old castrated male Shih Tzu dog (weighing $7.0 \mathrm{~kg}$ ) presented with major complaints of abrupt exercise intolerance and marked depression. According to the referring vet, the dog was treated for chronic dermatitis from demodecosis and otitis externa. At presentation, the dog showed pale mucosa and delayed capillary refill time $(>\sim 3.0$ $\mathrm{sec})$, suggesting anemia. His heart rate, rectal temperature, and systolic blood pressure were $120 \mathrm{bpm} / \mathrm{min}, 38.0^{\circ} \mathrm{C}$ and $135 \mathrm{mmHg}$, respectively. A routine complete blood cell count (CBC; IDEXX LaserCyte Dx Hematology Analyzer; IDEXX Laboratories, USA) revealed hypochromic regenerative anemia (hemoglobin $7.7 \mathrm{~g} / \mathrm{dL}$; reference range [RI], 12.4-19.1 g/ dL; hematocrit 22.2\%; RI, 29.8-57.5\%; reticulocyte $1.37 \%$;
RI, $0-1 \%$ ) and leukocytosis (white blood cells $26.3 \times \mathrm{K} / \mu \mathrm{L}$; RI, $5.4-15.3 \times \mathrm{K} / \mu \mathrm{L}$ ) with segment neutrophilia (neutrophils $23.3 \times \mathrm{K} / \mu \mathrm{L}$; RI, $1.2-6.8 \times \mathrm{K} / \mu \mathrm{L}$ ). A blood cytological examination revealed spherocytosis, anisocytosis, and polychromasia, indicating regenerative anemia (Fig. 1). A saline red blood agglutination test confirmed a positive reaction. Blood chemistry analysis (Vetscan; ABAXIS, USA) indicated increased blood urea (40 mg/dL; RI, 7-27 mg/dL) and total bilirubin $(1.6 \mathrm{mg} / \mathrm{dL}$; RI, $0-0.9 \mathrm{mg} / \mathrm{dL})$. Based on laboratory tests, the dog was tentatively diagnosed with IMHA and hospitalized for intensive therapy. The dog was initially treated with prednisolone ( $1 \mathrm{mg} / \mathrm{kg}$, per orally [PO], q12h, Solondo; Yuhan, Korea), azathioprine (2 mg/kg, PO, q24h, Imuran; Samil pharmaceuticals, Korea), danazol (5 mg/kg, PO, q12h, Danazol cap; Young Poong Pharmaceuticals, Korea), and ursodeoxycholic acid (15 mg/kg, PO, q24h, Ursa; Daewoong Pharmaceuticals, Korea). Despite intensive treatment with immunosuppressive drugs, the CBC performed on the next day showed worsening of anemia and leukocytosis (hematocrit 18.7\%; hemoglobin $6.2 \mathrm{~g} / \mathrm{dL}$; white blood cells $42.6 \times \mathrm{K} / \mu \mathrm{L}$ ). Therefore, a blood transfusion was performed with packed red blood cells $(112 \mathrm{~mL})$ to increase the dog's hematocrit levels. After the blood transfusion, the anemia improved (hematocrit $29 \%$; hemoglobin $9.4 \mathrm{~g} / \mathrm{dL}$ ), although leukocytosis deteriorated $(56.4 \times \mathrm{K} / \mu \mathrm{L})$. After the transfusion, the dog was administered medication including prednisolone $(2 \mathrm{mg} / \mathrm{kg}, \mathrm{PO}$,

*Corresponding author

Tel: +82-33-250-8681, Fax: +82-33-244-2367

E-mail: hyun5188@kangwon.ac.kr 


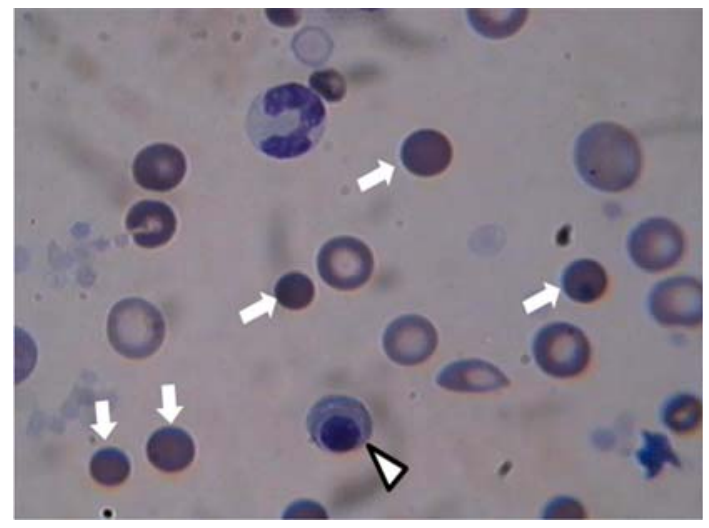

Fig. 1. Blood smear from this dog. There were numerous spherocytes (arrows), nucleated red blood cells (arrow head) due to immune-mediated hemolytic anemia. Diff-Quick stain. 1,000×.

q12h), azathioprine (2 mg/kg, PO, q24h), danazol (5 mg/kg, PO, q12h), famotidine (15 mg/kg, PO, q24h, Famotidine tab; Withus Pharmaceutical, Korea), biphenyl dimethyl dicarboxylate $(8.3 \mathrm{mg} / \mathrm{kg}$, PO, q24h, Lefotil tab; CMG Pharm, Korea), milk thistle extract $(35 \mathrm{mg} / \mathrm{head}, \mathrm{PO}, \mathrm{q} 24 \mathrm{~h}$, Silymarin Tab; Sinil pharmaceuticals, Korea), and ursodeoxycholic acid $(15 \mathrm{mg} / \mathrm{kg} /$ day, PO, q24h). After 20 days of pharmacotherapy, the dog still showed clinically significant anemia (hematocrit $19.4 \%$; hemoglobin $6.5 \mathrm{~g} / \mathrm{dL}$; white blood cells $45.6 \times$ $\mathrm{K} / \mu \mathrm{L})$. Therefore, azathioprine was replaced with cyclosporine $(7 \mathrm{mg} / \mathrm{kg}$, PO, q24h, Cypol; Chong Kun Dang pharmaceuticals, Korea). During treatment with cyclosporine, the dog started showing side effects (i.e., vomiting, depression, anorexia, or paleness) and more severe anemia (e.g., hematocrit 16.6\%; hemoglobin $5.4 \mathrm{~g} / \mathrm{dL}$ ). Blood chemistry also found increased renal and hepatic enzymes (blood urea $40 \mathrm{mg} / \mathrm{dL}$; alkaline phosphatase 2,028 U/L; RI, 20-150 U/L; alanine transaminase $412 \mathrm{U} / \mathrm{L}$; RI, $10-118 \mu / \mathrm{L}$ ). To improve his anemic condition, a blood transfusion was repeated with packed red blood cells $(80 \mathrm{~mL})$. Although the dog was less anemic after therapy (hematocrit 33.2\%; hemoglobin $11 \mathrm{~g} /$ $\mathrm{dL}$ ), his urine was suddenly cloudy. The urine was collected and analyzed. Further urinalysis, including a sediment test, revealed aciduria ( $\mathrm{pH}$ 6), leukocyturia (mostly neutrophils and macrophages), proteinuria $(3+)$, hemoglobinuria, and funguria (Fig. 2B). Fungal pseudohyphae were found in the urine and thus submitted for a fungal culture confirmation to a commercial veterinary lab (NEODIN Vetlab; Korea). To interrogate the extent of the urinary tract infection, an abdominal ultrasonographic examination was performed in the kidneys and urinary bladder. Ultrasonography revealed pelvic dilation, an irregularly thickened corticomedullary junction in the kidneys, and markedly increased echogenecity of the renal pelvis (Fig. 3A). The wall of the urinary bladder was intact but thickened (Fig. 3B). There was hyperechogenic sediment (arrow head) inside the bladder (Fig. 3B).

The tentative diagnosis was candidal cystitis and the dog was treated with intermittent bladder washing with Tris-EDTA
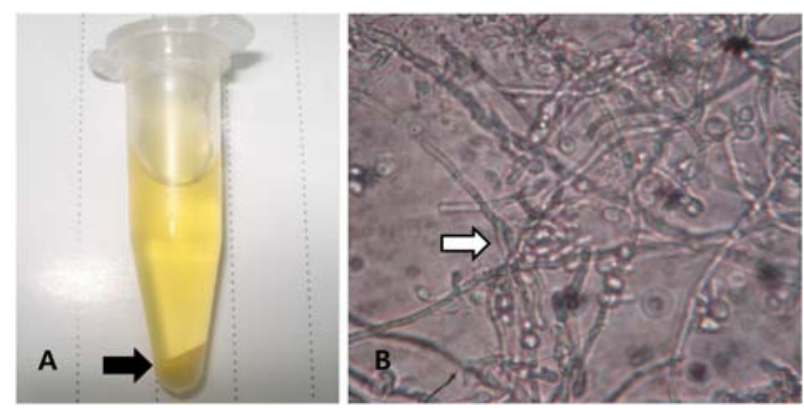

Fig. 2. Urine from this case. (A) Cloudy sediment (black arrow) after centrifugation of urine. (B) Microscopic examination found pseudohyphae and budding yeast (white arrow) indicating Candida infection. $1,000 \times$.
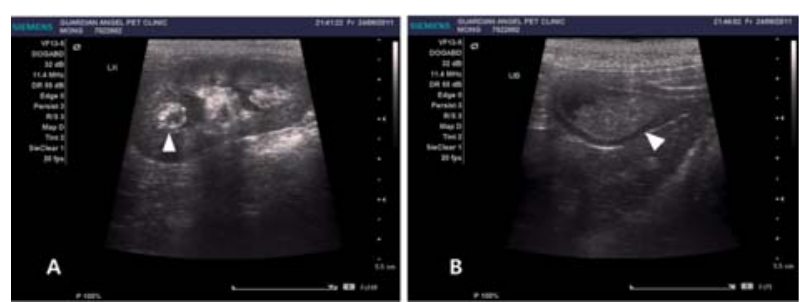

Fig. 3. Abdominal ultrasonography of this case. (A) There was pelvic dilation (arrow head) and irregularly thickened corticomedullary junction in the left kidney. The echogenecity of renal pelvis was markedly increased. (B) The wall of urinary bladder was intact but thickened. There was hyperechogenic sediment (arrow head) inside bladder.

solution (TrizEDTA; Dechra, USA) and with oral medications, including cephalexin (30 mg/kg, q12h, Cephalexin cap; KOLON Pharma, Korea), ketoconazole $(10 \mathrm{mg} / \mathrm{kg}, \mathrm{q} 12 \mathrm{~h}$, Spike tab; JW Pharmaceutical, Korea) and ursodeoxycholic acid (15 mg/kg, q24h). Cyclosporin was again replaced with azathioprine $(2 \mathrm{mg} / \mathrm{kg}$, PO, q24h). However, the dog died suddenly after 6 days of therapy at home. A postmortem examination was declined owing to refusal from the owners. A C. albicans infection was finally confirmed by a fungal culture 10 days after the dog's death.

This case report described a secondary yeast infection $(C$. albicans) due to immunosuppressive therapy against IMHA in a dog. Candida spp. are saprophytic yeasts. Three Candida species, including $C$. albicans, $C$. parapsilosis, and $C$. tropicalis, are pathogenic to dogs and occasionally colonize the skin, oral cavity, and urogenital, and gastrointestinal tracts in dogs [10]. Prolonged immunosuppression from several disease conditions can develop into candidiasis in dogs $[3,4$, $8,9]$. Even though infections can be confined to the skin, dogs with systemic candidiasis can present more general symptoms, including peritonitis and chronic cystitis [1], as seen in this case. Although one recent study has reported candiduria from chronic cystitis in a dog [1], this is the first case study describing secondary candidal cystitis from immunosuppressive therapy. Six Candida spp. were isolated in one 
recent retrospective study with 20 dogs and cats with candidal urinary infections. $C$. albicans was the most common species of candida isolated in this study. The pre-existing diseases in that study group were diabetes mellitus $(n=4)$, nonurogenital neoplasia $(n=3)$, and noncandidal urogenital disease $(n=14)$, while the medications were antibiotics $(n=16)$ and corticosteroids $(n=6)$. All animals had a certain degree of local or systemic immune suppression, owing to pre-existing diseases. This retrospective study concluded that proper management for pre-existing conditions should be performed prior to medical treatment against a candidal urinary infection [9]. The cause of our case might be long-term administration of steroid and immune suppressive drugs to control clinical signs related to IMHA. According to the literature [5], candidal UTIs in dogs and cats have rarely caused typical clinical signs related to a lower UTI, including dysuria, pollakiuria, and hematuria, but have occasionally caused yellow-whitish clotting in urine. Therefore, it often led to clinicians missing a potential candidal infection in animals with diabetes mellitus or extraneous steroid overdose. In this case, a diagnosis of candidal infection was made in a later stage of disease (after clinical signs had worsened) because the dog did not show any clinical signs related to a UTI. An accidental finding of cloudy urine forced the authors to perform urinalysis and a urine culture. An earlier intervention against candidal cystitis might result in a more favorable outcome after antifungal therapy in this case.

Although the recommended therapeutic protocol against candidal infections in dogs has yet been established, the Infectious Diseases Society of America (IDSA) has proposed several therapeutic protocols against human systemic candidal infection [2]. According to the IDSA, an intravenous injection of amphotericin $\mathrm{B}$ and an intravenous or oral administration of fluconazole were the most effective against systemic candidal infections in humans [9]. A therapy with fluconazole had fewer side effects than that with amphotericin B in humans [6]. Fluconazole has also been recommended for therapy of candidiasis in dogs $[5,8]$. Treatment with fluconazole is generally required for 2 to 3 weeks in dogs. One recent study reported that an intermittent bladder infusion with clotrimazole was successful for treatment of candiduria in a dog [4]. The authors expected a more favorable outcome if the fluconazole was used to treat a candidal infection in our case.
In conclusion, this case report described a secondary $C$. albicans UTI resulting from IMHA in a dog. Although a $C$. albicans UTI has been well documented in dogs and cats, a UTI associated with IMHA has never been reported in dogs. Since the clinical signs from a $C$. albicans UTI in dogs are mostly obscure, a regular routine urinalysis with a urine culture is required for dogs with immune suppression.

\section{References}

1. Choi US. [Practical Guide to Diagnostic Cytology of the Dog and Cat]. 1st ed. pp. 351, OKVET, Daejeon, 2012. Korean.

2. Edelmann A, Krüger M, Schmid J. Genetic relationship between human and animal isolates of Candida albicans. J Clin Microbiol 2005, 43, 6164-6166.

3. Forward ZA, Legendre AM, Khalsa HD. Use of intermittent bladder infusion with clotrimazole for treatment of candiduria in a dog. J Am Vet Med Assoc 2002, 220, 1496-1498.

4. Humphrey MJ, Jevons S, Tarbit MH. Pharmacokinetic evaluation of UK-49,858, a metabolically stable triazole antifungal drug, in animals and humans. Antimicrob Agents Chemother 1985, 28, 648-653.

5. Jin Y, Lin D. Fungal urinary tract infections in the dog and cat: a retrospective study (2001-2004). J Am Anim Hosp Assoc 2005, 41, 373-381.

6. Karlowsky JA, Zhanel GG, Klym KA, Hoban DJ, Kabani AM. Candidemia in a Canadian tertiary care hospital from 1976 to 1996. Diagn Microbiol Infect Dis 1997, 29, 5-9.

7. Manolakaki D, Velmahos G, Kourkoumpetis T, Chang, Y, Alam HB, De Moya MM, Mylonakis E. Candida infection and colonization among trauma patients. Virulence 2010, 1, 367-375.

8. Pappas PG, Rex JH, Sobel JD, Filler SG, Dismukes WE, Walsh TJ, Edwards JE. Guidelines for treatment of candidiasis. Clin Infect Dis 2004, 38, 161-189.

9. Pressler BM. Candidiasis and rhodotorulosis. In: Greene CE (ed.). Infectious Diseases of the Dog and Cat. 4th ed. pp. 666-671, Saunders, Philadelphia, 2012.

10. Pressler BM, Vaden SL, Lane IF, Cowgill LD, Dye JA. Candida spp. urinary tract infections in 13 dogs and seven cats: predisposing factors, treatment, and outcome. J Am Anim Hosp Assoc 2003, 39, 263-270.

11. Warren NG, Hazen KC. Candida, Cryptococcus, and other yeasts of medical importance. In: Murray PR, Baron EJ, Pfaller MA, Tenover FC, Yolken RH (eds.). Manual of Clinical Microbiology. 7th ed. pp. 1184-1199, ASM press, Washington DC, 1999. 